\title{
考虑间歇期的飞机连杆式舱门 收放机构竞争失效分析
}

\author{
刘敬一 ${ }^{1}$ ，张玉刚 ${ }^{2}$ ，庄新臣 ${ }^{3}$ ，庞欢 ${ }^{1}$
}

$\left(\begin{array}{l}\text { 1.长安大学 汽车学院, 陕西 西安 } 710064 ; \text { 2. 西北工业大学 航空学院, 陕西 西安 710072; } \\ \text { 3.清华大学 机械工程学院, 北京 } 100091\end{array}\right)$

\begin{abstract}
摘要: 建立了考虑间歇期的适用于飞机连杆式舱门收放机构的竞争失效模型。使用泊松过程、Archard 磨损模型和功能失效函数对飞机机构的突发型失效和退化型失效进行建模; 充分考虑飞机存在 不同工作状态的实际特点,引入间歇期因素建立了新的竞争失效模型; 以某型飞机连杆式舱门收放机 构作为案例, 在深入分析机构工作原理和失效特点的基础之上,对所建模型进行应用研究,结果表明 间歇期的引入使得各模式失效概率和系统可靠度呈现不同的演化特征,且考虑间歇期的竞争失效模 型具有良好的工程实用性。
\end{abstract}

\section{关 键 词: 竞争失效; 间歇期; 飞机连杆式舱门收放机构 \\ 中图分类号: V328.5 \\ 文献标志码: A \\ 文章编号: 1000-2758(2021 )05-1105-09}

连杆式舱门收放机构在飞机中应用广泛, 诸如 起落架舱门收放机构、货舱门收放机构等。该类机 构的失效会直接影响到飞机的安全性, 确保该类机 构的可靠运行尤为重要。本文所研究的某型飞机舱 门的连杆式收放机构,存在突发型失效和退化型失 效并存的现象, 机构系统的实际失效是此二类失效 相互竞争的结果。充分研究该机构的运行原理,结 合机构的运行具备间歇期这一实际特点建立竞争失 效模型, 可以更为准确地对该机构可靠度的演化进 行评估。

目前,在有关飞机机构的竞争失效研究方面, 侯 聿 $^{[1]}$ 研究了飞机起落架收放作动筒指型锁的磨损; 刘成业等 ${ }^{[2]}$ 针对某型民用飞机舱门锁结构变形现 象, 从接触力角度开展了失效研究。以连杆机构为 例, 印寅 ${ }^{[3]}$ 分析了某型起落架的动力学特性, 并对 起落架机构进行了可靠性研究; 郑怡 ${ }^{[4]}$ 基于多体动 力学, 分析了发动机中连杆机构的动态应力, 对连杆 失效造成发动机的破坏性故障进行了研究。郭庆、 刘晓娟等 ${ }^{[5-6]}$ 分别研究了航空发动机的竞争失效行
为, 从寿命预测、可靠性评估和性能退化等不同角度 对航空发动机的可靠性进行了研究。对于构件数目 较多的连杆机构而言, 各个构件的尺寸误差和运动 副中的间隙会产生较大的累计误差, 容易造成运动 精度的不足,但当前有关飞机机构竞争失效的研究 尚未涉及连杆类机构运动精度不足的问题。

在有关竞争失效模型的研究方面, Rafiee 等 ${ }^{[7]}$ 认为外界冲击的出现会引起退化过程退化速率的改 变,并综合多种因素建立了的竞争失效模型。Qiu 等 ${ }^{[8]}$ 考虑退化过程的 2 个阶段建立了竞争失效模 型, 并基于此分析了输油管道的可靠性。Y Yang 等 ${ }^{[9]}$ 研究了处于竞争失效模式影响下的油气输送管道的 维修保障策略。Hao 等 ${ }^{[10]}$ 对处于局部放电失效和 漏气失效 2 种失效模式影响下的气体绝缘输电保护 装置进行了研究, 建立了竞争失效模型。齐佳 ${ }^{[11]}$ 建 立并用试验验证了性能退化自恢复产品的相关竞争 失效模型。逯程 ${ }^{[12]}$ 等则研究了竞争失效条件下对 装备的选择性维修方法, 考虑多种维修方式对处于 多失效模式竞争作用下的系统进行了维修方式选择 
的综合优化。总的看来, 现有的竞争失效模型并未 考虑实际工作中 “工作期” 和 “间歇期” 相互结合的 特点, 需要建立新的模型。综上, 本文引人间歇期因 素, 建立了适用于飞机机构的竞争失效模型, 利用泊 松过程、Archard 磨损模型分别对突发型失效和退化 型失效进行研究; 利用所建模型, 对某型飞机连杆式 舱门收放机构的结构组成、工作原理和失效行为进 行深人研究, 结果证明所建模型能够有效地应用于 该机构的竞争失效分析, 同时可以推广至其他具有 相同特点的机械机构系统的研究之中。

\section{1 基本描述}

间歇期是区别于工作期的机构工作时间段,在 此时间内机构面临不同的环境特点, 机构自身的性 能退化速率也会发生变化。飞机机构通常处于两类 失效模式相互竞争的影响之下, 一类为退化失效 (例如机械轴的磨损、部件的老化), 一类为因外界 冲击 (例如意外的过大的工作载荷、振动冲击引起 的载荷等) 导致的突发失效。对于突发失效,使用 极限冲击模型描述, 即一旦有外界冲击超过了系统 的可承受量值, 则发生突发失效; 对于退化失效, 使 用考虑随机参数的通用路径模型描述, 当退化量累 计至一定量的时候, 系统发生退化失效。考虑间歇 期的系统竞争失效过程如图 1 所示。

图 1 时间轴中实线部分表示机构的工作期。当 系统处于工作期的时候, 退化量在较为快速地增加, 同时工作期期间会有一定的概率出现外界冲击 (例 如图中 $T_{1}$ 时间段内的 $W_{1}$ 和 $W_{2}$ ), 也就是说在工作 期期间, 系统会有一定的概率发生失效。每一次工 作期的时长定义为 $T_{1}$, 它的取值可取一个恒定值或 随机量值。图 1 时间轴的虚线部分表示机构的间歇 期,间歇期的意义为在此期间系统停止工作。处于 间歇状态系统的退化量因系统的 “间歇” 而增速减 缓, 同时 “间歇” 状态的系统不会受到外界冲击 (例 如图中 $T_{2}$ 时间段并没有任何外界冲击出现)。每一

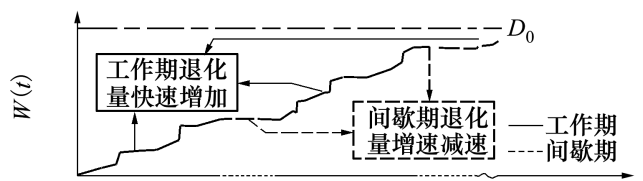

a) 退化失效过程

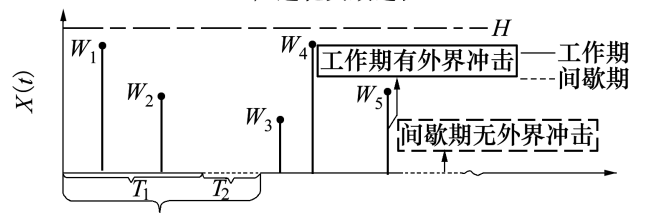

b) 突发失效过程

图 1 考虑间歇期的竞争失效过程

次间歇期的时长定义为 $T_{2}$, 其可取一个恒定值或随 机量值。相邻的一个工作期和一个间歇期定义为一 次工作循环( 图中 $T_{1}$ 时间段和 $T_{2}$ 时间段即可组合成 一次工作循环), 系统的寿命就是由若干个连续不 断的工作循环组成。

\section{2 建模过程}

\section{1 突发失效建模}

基于极限冲击模型对突发失效进行描述, 即当 某次外界冲击的量值大于系统的阈值之时, 系统发 生突发失效, 因而系统在外界冲击影响下的生存函 数表示

$$
P\left(W_{i}<H\right)=F_{W_{i}}(H) \quad i=1,2,3
$$

式中: $W_{i}$ 为在时间 $t_{i}$ 所发生的外界冲击的大小; $H$ 是系统突发失效阈值, 代表抵御外界冲击的能力。 考虑一种特殊情况, 如果外界冲击和系统阈值皆是 符合正态分布的随机变量, 上述生存函数表达式 变为

$$
F_{W_{i}}(H)=\Phi\left(\frac{\mu_{H}-\mu_{W_{i}}}{\sqrt{\sigma_{H}^{2}+\sigma_{W_{i}}^{2}}}\right)
$$

式中: $\mu$ 和 $\sigma$ 表示均值和方差, 即有 $H \sim N\left(\mu_{H}, \sigma_{H}^{2}\right)$ 和 $W_{i} \sim N\left(\mu_{W_{i}}, \sigma_{W_{i}}^{2}\right)$ 。考虑一系列的工作循环和间 歇期, 系统不发生突发失效的概率可表示为

$$
R_{H}(t)=P\left(\left\{W_{1}<H, \cdots, W_{i}<H\right\}\right)=
$$

$$
\left\{\begin{array}{l}
\sum_{i=0}^{\infty} P\left(\left\{W_{i}<H, \cdots, W_{i}<H\right\} \mid N\left(t-(n-1) T_{2}\right)=i\right) \times P\left(N\left(t-(n-1) T_{2}\right)=i\right) \\
\text { 工作期, 即当 }(n-1)\left(T_{1}+T_{2}\right)<t \leqslant n T_{1}+(n-1) T_{2},(n=1,2, \cdots) \\
\sum_{i=0}^{\infty} P\left(\left\{W_{i}<H, \cdots, W_{i}<H\right\} \mid N\left((n-1) T_{1}\right)=i\right) \times P\left(N\left((n-1) T_{1}\right)=i\right) \\
\text { 间歇期, 即当 } n T_{1}+(n-1) T_{2}<t \leqslant n\left(T_{1}+T_{2}\right),(n=1,2, \cdots)
\end{array}\right.
$$


式中: $n$ 表示工作循环的次数; $T_{1}$ 和 $T_{2}$ 分别表示一 次工作期和间歇期的持续时长。当 $n$ 取固定值的时 候,公式(3) 中的表达式( I ) 的取值会随着 $t$ 而发 生变化, 这是因为工作期期间系统会受到外界随机 冲击的影响, 有可能发生突发失效, 具体的概率值会 随着时间发生变化; 而表达式 ( II ) 则会保持定值, 这是因为间歇期并未有外界冲击发生, 系统的生存 概率等于上一个工作期最后时刻的生存概率。

\section{2 退化失效建模}

$$
X(t)=\left\{\begin{array}{c}
\varphi+\gamma_{1}\left(t-(n-1) T_{2}\right)+\gamma_{2}(n-1) T_{2},(n=1,2, \cdots) \\
\quad \text { 当 }(n-1)\left(T_{1}+T_{2}\right)<t \leqslant n T_{1}+(n-1) T_{2} \\
\varphi+\gamma_{1}\left(n T_{1}\right)+\gamma_{2}\left(t-n T_{1}\right),(n=1,2, \cdots) \\
\left.\quad \text { 当 } n T_{1}+(n-1) T_{2}\right)<t \leqslant n\left(T_{1}+T_{2}\right)
\end{array}\right.
$$

式中: $\varphi$ 为退化量的初值; $\gamma 1$ 和 $\gamma 2$ 分别表示工作期 和间歇期退化量的增加速率, 三者一般是符合特定

分布的随机变量。而因外界冲击所导致的额外退化 可表示为:

$$
S(t)=\left\{\begin{array}{l}
\left.\sum_{i=1}^{N\left(t-(n-1) T_{2}\right)} Y_{i},(n=1,2, \cdots), \text { 当 }(n-1)\left(T_{1}+T_{2}\right)<t \leqslant n T_{1}+(n-1) T_{2}\right) \\
\sum_{i=1}^{N\left((n-1) T_{1}\right)} Y_{i},(n=1,2, \cdots), \text { 当 } n T_{1}+(n-1) T_{2}<t \leqslant n\left(T_{1}+T_{2}\right)
\end{array}\right.
$$

式中, $N(t)$ 是一计数过程, 表示截止时刻 $t$ 所发生

合上述两表达式, 系统总的退化量表示为 的冲击的次数, 使用速率为 $\lambda$ 泊松过程来描述。综

$$
X_{s}(t)=\left\{\begin{array}{c}
\varphi+\gamma_{1}\left(t-(n-1) T_{2}\right)+\gamma_{2}(n-1) T_{2}+\sum_{i=1}^{N\left(t-(n-1) T_{2}\right)} Y_{i} \\
\text { 当 }(n-1)\left(T_{1}+T_{2}\right)<t \leqslant n T_{1}+(n-1) T_{2} \\
\varphi+\gamma_{1}\left(n T_{1}\right)+\gamma_{2}\left(t-n T_{1}\right)+\sum_{i=1}^{\left.N(n-1) T_{1}\right)} Y_{i} \\
\text { 当 } \left.n T_{1}+(n-1) T_{2}\right)<t \leqslant n\left(T_{1}+T_{2}\right)
\end{array}\right.
$$

如果系统不发生退化失效, 则要求总的退化量 不超过系统的许用阈值, 此时的生存概率表示为 $R_{X}(x, t)=P\left(X_{S}(t)<x\right)=$

$$
\sum_{i=0}^{\infty} P(X(t)+S(t)<x \mid N(t)=i) P(N(t)=i)
$$

基于卷积, 上述表达式进一步推导为

$$
R_{X}(x, t)=
$$

$$
\sum_{i=0}^{\infty}\left\{\left(\int_{0}^{x} G(x-u, t) f_{Y}^{\langle i\rangle}(u) \mathrm{d} u\right) \times \frac{\exp (-\lambda t)(\lambda t)^{i}}{i !}\right\}
$$

式中, $x$ 为退化阈值, 在本节中退化阈值的取值为 $D_{0}, G(x, t)$ 是总退化量 $X(t)$ 的累积分布函数, $f_{Y}^{\langle i\rangle}$ 为 $i$ 个独立同分布变量 $Y_{i}$ 的和的概率密度函数。类 似地, 考虑一种特殊情况, 当每次外界冲击所导致的
额外退化量 $Y_{i}$ 和退化速率 $\gamma$ 服从正态分布时 (即 $Y_{i}$ $\left.\sim N\left(\mu_{Y_{i}}, \sigma_{Y_{i}}^{2}\right), \gamma \sim N\left(\mu_{\gamma}, \sigma_{\gamma}^{2}\right), \gamma_{2} \sim N\left(\mu_{\gamma_{2}}, \sigma_{\gamma_{2}}^{2}\right)\right)$, 上述表达式可以进行进一步地推导简化。

\section{3 考虑间歇期的竞争失效系统可靠性及其演化 建模}

根据对受多相关竞争失效模式影响下系统的定 义, 系统有可能发生突发失效也有可能发生退化失 效,任一种失效模式的发生都会导致系统的失效, 因 而只有当所有的冲击都在系统的承受范围之内且退 化量值尚未超过许用國值的时候, 系统才能保持正 常。当每次外界冲击所导致的额外退化量 $Y_{i}$ 和退 化速率 $\gamma_{i}$ 服从正态分布时 (即有 $Y_{i} \sim N\left(\mu_{Y}, \sigma_{Y}^{2}\right), \gamma_{1}$ $\left.\sim N\left(\mu_{\gamma_{1}}, \sigma_{\gamma_{1}}^{2}\right), \gamma_{2} \sim N\left(\mu_{\gamma_{2}}, \sigma_{\gamma_{2}}^{2}\right)\right)$, 机构的可靠度 表示为公式 $(10)$ 。 


$$
\begin{aligned}
& R_{\text {sys }}(t)=P\left(W_{1}<H, W_{2}<H, \cdots, X_{S}(t)<D_{0}\right)= \\
& \sum_{i=0}^{\infty} P\left\{\left(W_{1}<H, \cdots, W_{i}<H, X(t)+S(t)<D_{0}\right) \mid N(t)=i\right\} P(N(t)=i)= \\
& \sum_{i=0}^{\infty}\left\{\begin{array}{l}
\left.F_{W}^{i}(H) \Phi\left(\frac{x-\left(\mu_{\gamma_{1}}\left(t-(n-1) T_{2}\right)+\mu_{\gamma_{2}}(n-1) T_{2}+\varphi+i u_{Y_{i}}\right)}{\sqrt{\left.\sigma_{\gamma_{1}}^{2}\left(t-(n-1) T_{2}\right)^{2}+\sigma_{\gamma_{2}}^{2}(n-1) T_{2}\right)^{2}+i \sigma_{Y_{i}}^{2}}}\right)\right], \\
\text { 工作期, 即当 }(n-1)\left(T_{1}+T_{2}\right)<t \leqslant n T_{1}+(n-1) T_{2} \\
\exp \left(-\lambda\left(t-(n-1) T_{2}\right)\right)\left(\lambda\left(t-(n-1) T_{2}\right)\right)^{i} \\
\sum_{W}^{i}(H) \Phi\left(\frac{x-\left(\mu_{\gamma_{1}}\left(n T_{1}\right)+\mu_{\gamma_{2}}\left(t-n T_{1}\right)+\varphi+i u_{Y_{i}}\right)}{\sqrt{\sigma_{\gamma_{1}}^{2}\left(n T_{2}\right)^{2}+\sigma_{\gamma_{2}}^{2}\left(t-n T_{1}\right)^{2}+i \sigma_{Y_{i}}^{2}}}\right) \\
\sum_{i=0}^{\infty}\left\{\begin{array}{l}
\exp \left(-\lambda\left(\left(n T_{1}\right)\right)\left(\lambda\left(n T_{1}\right)\right)^{i}\right. \\
i !
\end{array}\right\},
\end{array}\right.
\end{aligned}
$$

间歇期, 即当 $\left.n T_{1}+(n-1) T_{2}\right)<t \leqslant n\left(T_{1}+T_{2}\right)$

\section{3 某型飞机连杆式舱门收放机构竞争 失效研究}

\section{1 机构工作原理及失效模式}

图 2 为该机构的收放过程示意图。机构可以划 分为作动筒、连杆机构和舱门三大部分。作动筒为

动力源组件为整个机构提供动力 $;$ 连杆机构承担连 接和传动的作用; 舱门属于输出组件, 在动力源和连 杆的带动之下, 实现打开和关闭状态的机构功能。 该收放机构的功能要求在既定的时间内完成舱门的 打开和关闭动作，由于作动筒性能退化和运动副间 隙演化,导致该机构存在 2 个失效模式:

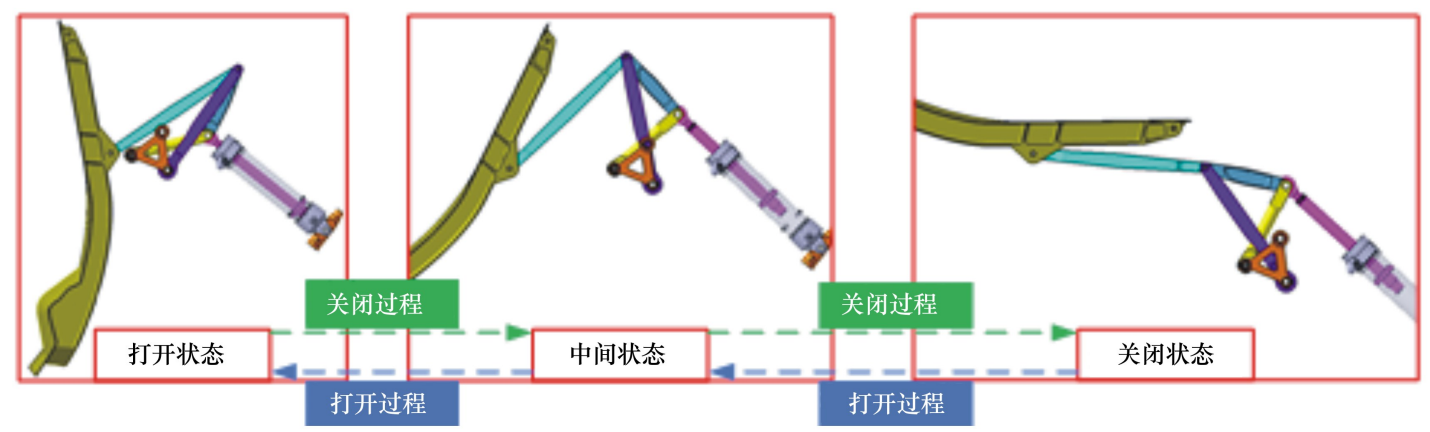

图 2 连杆式舱门收放机构功能示意

失效模式 1 : 作动筒堵塞导致的驱动力下降。 作动筒失效的最主要原因之一是因液压油中异物导 致的阻塞 ${ }^{[13]}$, 进而导致作动筒可输出液压力下降, 从而造成机构的失效。根据文献 [14]可知, 导致堵 塞的异物来源可能是外界环境带人,也可能是由于 作动筒筒壁和活塞杆之间的摩擦造成, 这个过程可 以用冲击模型来描述。图 3 为该失效模式示意图, 属于突发失效;

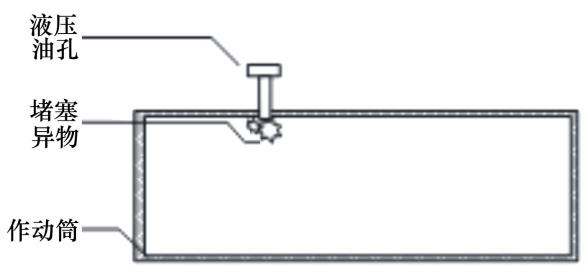

图 3 机构失效模式 1 
筒驱动一系列的连杆运动达到关闭位置, 只有达到 满足要求的既定位置, 舱门的关闭功能才可视为完 成。但由于随机性和磨损的存在, 随着使用次数和 时间的延长, 运动副的间隙会增大, 进而有可能导致 舱门无法关闭到位, 当舱门的实际位置的转角和要 求转角之间的误差超过一定程度的时候, 就可视为 功能无法完成。图 4 为该失效模式示意图, 属于可 退化的功能失效。

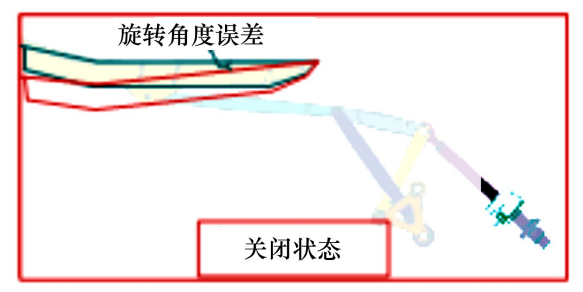

图 4 机构失效模式 2

\section{2 考虑间歇期的连杆式舱门收放机构竞争失效 建模}

\subsection{1 突发型失效建模}

导致作动筒堵塞异物尺寸的大小和出现时间都 是随机的, 可以把异物看作是量值和到来时间均不

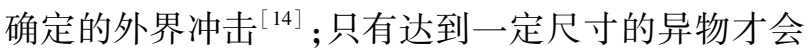
导致堵塞的发生。以 $W_{i}$ 表示出现在液压油中异物 的尺寸大小, $H$ 表示能够造成作动筒堵塞的临界尺 寸值,该机构不发生突发失效的概率表示为:

$$
P\left(W_{i}<H\right)=F_{W_{i}}(H) \quad i=1,2,3, \cdots
$$

式中, $W_{i}$ 是独立同分布 (independent identically distributed) 的变量, 表示每次出现异物的随机尺寸值。 使用泊松过程表示异物的随机出现时刻, 综上, 机构 不发生突发失效的概率表示为

$$
\begin{aligned}
& R_{X}=\sum_{i=0}^{\infty} R(t \mid N(t)=i) P(N(t)=i)= \\
& \quad \sum_{i=0}^{\infty} P\left\{\left(W_{1}<H, \cdots, W_{i}<H\right) \mid N(t)=i\right\} \times \\
& P(N(t)=i)
\end{aligned}
$$

式中: $N(t)$ 表示泊松过程, 用于衡量异物出现时刻 的不确定性; $\lambda$ 为泊松过程的速率, 表示异物出现的 频繁程度, 速率越大则出现异物的频率越高。不发 生突发失效要求没有异物出现 (即式中 $i$ 取值为 0 ) 或出现的异物尺寸皆小于阈值 $H$ 。

\subsection{2 退化型失效建模}

1）连杆有效长度的表示

图 5 为连杆机构两杆件之间的铰链式运动副间
隙随磨损而变化的简化示意图。实线、点划线和虚 线分别表示初始时刻 $t_{0}$ 、中间时刻 $t_{1}$ 和最终状态 $t_{2} 3$ 个时间点销轴和套筒的尺寸变化 (为了示意图的展 示效果, 尺寸进行了相应的放大), 随着时间的演 化, 销轴和套筒因磨损发生了尺寸的改变, 运动副间 隙 $C_{0}, C_{1}$ 和 $C_{2}$ 也逐渐增大。

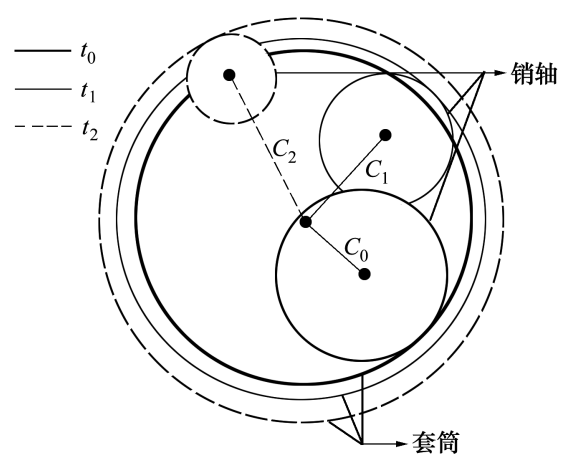

图 5 铰链式运动副间隙增大示意图

根据等效长度理论 ${ }^{[15]}$, 机构连杆参与运动的等 效长度由连杆本身尺寸长度、间隙尺寸和连杆所受 拉压状态共同决定。等效杆长理论可简单表述为, 参与机构运动传递的连杆等效长度等于连杆的实际 长度加上 (减去) 间隙长度, 表示为 $l^{\prime}=l \pm C=l \pm$ $\left(R_{1}-R_{2}\right)$, 其中 $l$ 为连杆的实际几何长度, $l^{\prime}$ 为连杆 参与运动传递的有效长度, $C$ 为间隙的尺寸, $R_{1}$ 和 $R_{2}$ 分别表示销轴和套筒的半径。

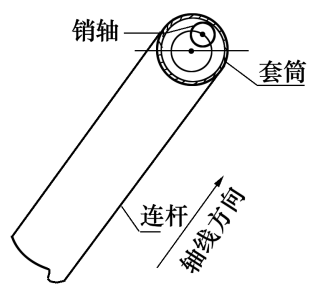

a) 受拉杆

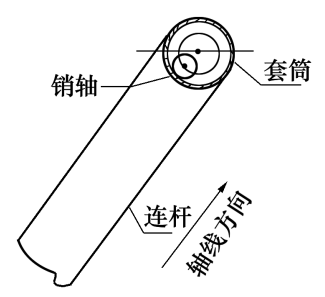

b）受压杆
图 6 连杆有效长度示意图

如图 6 所示, 当连杆处于受拉状态的时候, 实际 参与运动传递的长度会因间隙的存在而增大, 有效 长度表示为 $l+C$, 类似地, 当连杆受压时, 有效长度 会因间隙的存在而减小, 表示为 $l-C$ 。

2) 运动副磨损及间隙演化建模

连杆机构铰链磨损是影响传动精度的重要因 素,Archard 模型 ${ }^{[16]}$, 可以对磨损体积、滑动速度、垂 直载荷以及材料硬度等参数进行解释, 该模型已经 
得到了广泛的认可和应用。Archard 磨损模型的计 算公式表示为:

$$
\frac{h}{s}=\frac{k p}{H}
$$

式中: $h$ 表示磨损深度, 当考虑均匀磨损的时候 $h$ 等 同于间隙 $C$ 的变化量; $s$ 为相对滑移距离, $k$ 为无量纲 的磨损系数, $H$ 为较软材料的硬度, $p$ 为接触应力, $E$ 为材料相应的杨氏模量。接触应力的大小可以根据 赫兹接触理论计算求得, 即

$$
p=0.5642 \sqrt{\frac{F\left(R_{2}-R_{1}\right)}{R_{2} R_{1}\left(\frac{1-v_{1}^{2}}{E_{1}}+\frac{1+v_{2}^{2}}{E_{2}}\right)}}
$$

式中: $F$ 为单位长度的载荷; $R_{1}$ 和 $R_{2}$ 为销轴和衬套 的半径; $v$ 为材料的泊松比。

相对滑移距离可以根据几何关系推导得到, 即

$$
s=2 \pi r \frac{\varphi}{360^{\circ}} n
$$

式中: $\varphi$ 为一个运动周中销轴和衬套的相对转角; $n$ 为运动的周期数。

综合上述各表达式,磨损的深度可以计算为

$$
h=n \int_{0}^{t} \frac{k p(t) \omega(t)}{360^{\circ} b H} \mathrm{~d} t
$$

式中: $p(t)$ 和 $\omega(t)$ 分别为接触应力和转动角度随 时间的函数, 可以通过机构的动力学分析得到。根 据有效长度理论, 间隙的演化使得连杆参与运动的 有效长度发生改变。

3 ) 机构功能失效建模研究功能失效的表现形 式为 “舱门无法关闭到位”, 选取舱门从打开位置到 关闭位置时候所转过的角度作为功能表征量, 根据 舱门工作要求, 满足规定的旋转角度为 $A_{0}=120^{\circ}$ 。 为了提高计算效率, 使用一阶响应面构造舱门的功 能表征函数,构造步骤如下:

step1 依据运动原理搭建参数化机构动力学 仿真模型;

step2 按照各输人参数的分布 (见表 $1 \sim 3$ ) 进 行拉丁超立方抽样, 将抽样值代人仿真模型, 得到成 组的输人输出;

step3 利用 step2 中得到的结果进行响应面的 构造并进行精度验证。

构造得到的连杆式舱门收放机构功能表征量的 响应面函数为:

$$
A(t)=-89.7+0.41 \times l_{E F}(t)+0.49 \times l_{C E}(t)-
$$

$$
0.00049 \times l_{A B}(t)
$$

式中: $A(t)$ 为舱门的关闭角度; $l(t)$ 表示间隙的退 化影响连杆的有效长度。当舱门实际旋转的角度 $A(t)$ 和要求角度 $A_{0}$ 的偏差超过许用偏差 $\delta$ 的时候, 舱门发生功能退化失效。因此, 舱门的功能满足要 求即不发生功能退化失效的公式表达为:

$$
R_{F}(t)=P\left(\left|A(t)-A_{0}\right|<\delta\right)
$$

式中: $A_{0}$ 为理想状态舱门关闭时所旋转的角度; $A(t)$ 为舱门实际所旋转的角度, 其具体的取值由功 能表征函数计算决定; $\delta$ 为许用误差。

\subsection{3 机构可靠度及其演化规律}

若要该连杆式收放机构不发生失效, 则需突发 失效和功能退化失效都不能发生, 综合公式 (12) 和 公式(18), 推导得到机构的整体可靠度为

$$
\begin{gathered}
R_{\mathrm{sys}}(t)=\sum_{i=0}^{\infty} P\left(\left(\begin{array}{l}
\left(\left|A(t)-A_{0}\right|<\delta\right), \\
\left(W_{1}<H, \cdots, W_{n}<H\right.
\end{array}\right) \mid N(t)=n\right) \times \\
P(N(t)=n)=P\left(A_{0}-\delta<A(t)<A_{0}+\delta\right) \times \\
\sum_{i=0}^{\infty} F_{W_{i}}^{n}(H) \times \frac{\exp (-\lambda t)(\lambda t)^{n}}{n !}
\end{gathered}
$$

特殊的, 若舱门的实际旋转角度 $A(t)$ 服从正态分布 , 上述表达式进一步推导为:

$$
\begin{gathered}
R_{\mathrm{sys}}(t)=\left[\Phi\left(\frac{A_{0}+\delta-\left(\mu_{A}\right)}{\sqrt{\sigma_{A}^{2}}}\right)-\Phi\left(\frac{A_{0}-\delta-\left(\mu_{A}\right)}{\sqrt{\sigma_{A}^{2}}}\right)\right] \times \\
\sum_{n=0}^{\infty} F_{W_{i}}^{n}(H) \times \frac{\exp (-\lambda t)(\lambda t)^{n}}{n !}
\end{gathered}
$$

\section{3 计算结果}

图 7 为该连杆收放机构的简化示意图, 结合舱 门关闭状态各个连杆的拉压状态, 各连杆的有效长 度如表 1 所示。连杆式舱门收放机构并非总处于运 动过程之中, 而是会有一段时间的间歇状态。由于 相对运动的停止, 处于间歇时的连杆式舱门收放机 构不会发生进一步的磨损。结合实际的工况, 该连 杆式舱门收放机构每有 40000 次开闭 (即工作状态

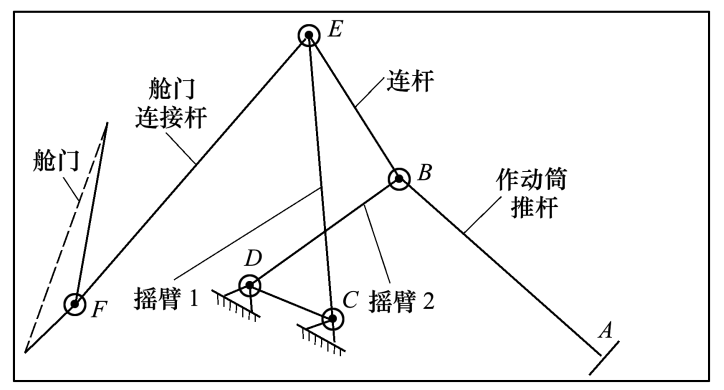

图 7 连杆式舱门收放机构简化示意图 
表 1 舱门收放机构连杆状态

\begin{tabular}{cccc}
\hline 连杆名称 & 受力状态 & 质量 $/ \mathrm{kg}$ & 有效长度 $l_{x x}(t)$ \\
\hline 作动筒推杆 $L_{A B}$ & 受压 & 3.34 & $l_{A B}-C_{B}(t)$ \\
摇臂 $1 L_{B D}$ & 受压 & 1.126 & $l_{B D}-C_{D}(t)$ \\
连杆 $L_{B E}$ & 受压 & 0.962 & $l_{B E}-C_{E}(t)$ \\
摇臂 $2 L_{C E}$ & 受拉 & 2.04 & $l_{C E}+C_{C}(t)$ \\
舱门连接杆 $L_{E F}$ & 受压 & 2.275 & $l_{E F}-C_{F}(t)$ \\
\hline
\end{tabular}

的等效时长) 就会有 10000 次等效时长 (即间歇状 态的等效时长) 的间歇状态, 当机构处于间歇状态 时, 各个运动副的磨损不再随时间增加。根据公式 (13) (16), 可以计算得到连杆式舱门收放机构各 铰链的磨损规律, 参数的含义和取值如表 2 所示。

表 2 舱门收放机构磨损相关参数

\begin{tabular}{ccc}
\hline 名称 & 符号 & 参数取值 \\
\hline 磨损系数 $/\left(\mathrm{mm}^{3} \cdot \mathrm{Nm}^{-1}\right)$ & $k$ & $1.736 \times 10^{-4}$ \\
轴颈宽度 $/ \mathrm{mm}$ & $b$ & 40 \\
衬套初始半径 $/ \mathrm{mm}$ & $R_{1}$ & 50 \\
轴颈初始半径 $/ \mathrm{mm}$ & $R 2$ & 49.9 \\
衬套材料泊松比 & $v_{1}$ & 0.29 \\
轴颈材料泊松比 & $v_{2}$ & 0.38 \\
衬套材料杨氏模量 $/ \mathrm{GPa}$ & $E_{1}$ & 0.139 \\
轴颈材料杨氏模量 $/ \mathrm{GPa}$ & $E_{2}$ & 206 \\
\hline
\end{tabular}

图 8 为各个铰链间隙磨损量的演化示意图。图 中纵坐标为磨损深度, 横坐标为使用舱门收放机构 的开闭次数所表示的等效时间。

将表 3 参数带人公式 $(12) \sim(20)$, 可以计算得 到该连杆式舱门收放机构各失效模式的失效概率 (见图 9) 和系统可靠度及其演化规律 (见图 10)。 从图 9 看出,等效工作时间为 21930 次时,2 种模式 的失效概率相同，而不考虑间歇期情况下 2 种模式 失效概率相同时对应的等效工作时间约提早至 2000 次。从图 10 可以看出, 考虑间歇期后的收放 机构其退化速率降低, 可靠度演化速率随之减缓。 在等效工作时间为 21930 次时, 相对应的可靠度约 相差 $20 \%$ 。

\section{表 3 连杆式舱门收放机构参数取值}

\begin{tabular}{cccc}
\hline 参数 & 参数含义 & 取值 & 备注 \\
符号 & & & \\
\hline$l_{A B}{ }^{\prime}$ & 作动筒推杆 $A B$ 的几何尺寸 & $\sim N(476.3,4.72)$ & 受压 \\
$\mathrm{mm}$ & & & \\
$l_{B D}^{\prime}$ & 摇臂 $B D$ 的几何尺寸 & $\sim N(280.5,2.82)$ & 受压 \\
$\mathrm{mm}$ & & & \\
$l_{B E} /$ & 连杆 $B E$ 的几何尺寸 & $\sim N(260,2.62)$ & 受压 \\
$\mathrm{mm}$ & & $\sim N(440,4.42)$ & 受拉 \\
$l_{C E} /$ & 摇臂 $2 C E$ 的几何尺寸 & \\
$\mathrm{mm}$ & & & \\
$l_{E F} /$ & 舱门连接杆 $E F$ 的几何尺寸 & $\sim N(545,5.52)$ & 受压 \\
$\mathrm{mm}$ & 突发失效國值 & & - \\
$H / \mathrm{cm}$ & 泊松过程速率 & $2.5 \times 10^{-5}$ & - \\
$\lambda$ & & & \\
\hline
\end{tabular}

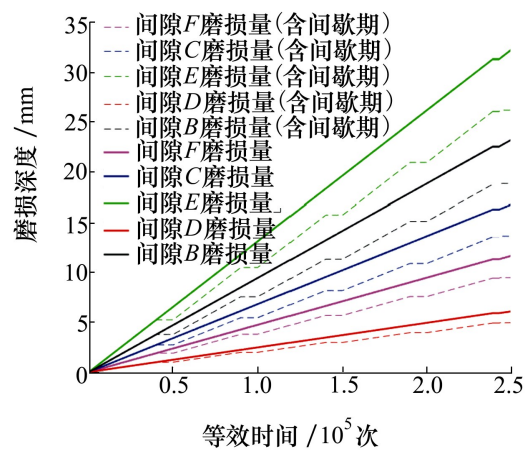

图 8 各处间隙磨损量演化示意图

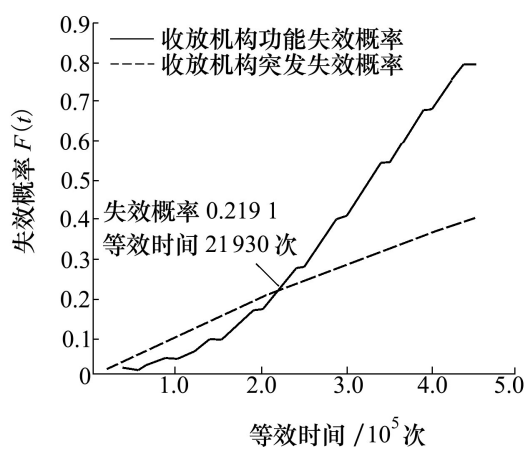

图 9 失效概率演化示意图

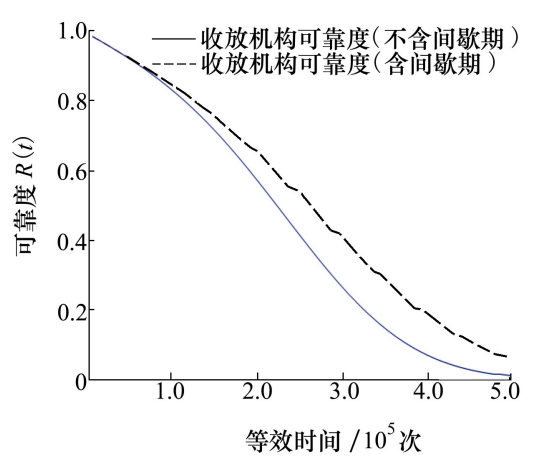

图 10 连杆式舱门收放机构 可靠度演化示意图 


\section{4 结 论}

本文建立了考虑间歇期的竞争失效模型, 并以 某型飞机连杆式舱门收放机构为研究对象, 分析了 其可靠度演化规律。连杆式收放机构处于突发失效 (因作动筒突然堵塞导致) 和功能退化失效 (因运动 副间隙演化导致的运动精度不足) 2 种失效模式的 竞争作用之下, 基于本文研究, 做出如下结论和 展望：

1) 机构铰链磨损并非直接导致机构失效, 而是 产生的误差通过功能表征函数进一步传递至功能表
征量, 引起旋转角度不符合要求引起功能失效;

2) 间歇期的存在改变了各个铰链间隙的磨损 过程,使得 2 种模式失效概率相等所对应的等效时 间由约 2000 次延长至 21930 次。在此时刻后, 功 能失效成为舱门收放机构的主要失效模式, 需在维 护保养工作中着重关注;

3 ) 间歇期使得机构可靠度退化减缓, 在等效时 间为 21930 次时,减缓程度约 20\%;

4) 本文所建考虑间歇期的竞争失效模型可推 广至其他具有间歇期特征的系统可靠性分析中,例 如运载车辆传动系统、电脑存储系统等。

\section{参考文献:}

[1] 侯聿. 飞机起落架收放作动筒指型锁的磨损研究 [D ]. 南京: 南京航空航天大学, 2016

HOU Yu. Research on the wear of aircraft landing gear retract actuator lock[D]. Nanjing: Nanjing University of Aeronautics and Astronautics, 2016 (in Chinese)

[2] 刘成业, 黄振庭, 周颖. 民用飞机舱门飞行锁机构接触力计算分析 [ J ] . 航空工程进展, 2018, 9(1): 91-98

LIU Chengye, HUANG Zhenting, ZHOU Ying. Calculation analysis on the door's contact force of the flight lock mechanism of civil airplane $[\mathrm{J}]$. Advances in Aeronautical Science and Engineering, 2018, 9(1): 91-98 (in Chinese)

[3] 印寅. 起落架收放动力学及可靠性研究 [D]. 南京: 南京航空航天大学, 2017

YIN Yin. Dynamics and reliability analysis of retraction of landing gear[D]. Nanjing: Nanjing University of Aeronautics and Astronautics, 2017 (in Chinese)

[4] 郑怡. 基于多体动力学的发动机连杆动态应力计算 $[D]$. 重庆: 重庆大学, 2016

ZHENG Yi. Dynamic stress calculation of engine connecting rod based on multi-body dynamics[D]. Chongqing: Chongqing University, 2016 (in Chinese)

[5] 郭庆, 徐甘生, 赵洪利. 基于蒙特卡洛发动机竞争失效的下发仿真模型 [ J ]. 航空动力学报, 2019, 34(3): 616-626 GUO Qing, XU Gansheng, ZHAO Hongli. Monte carlo-based competitive failure delivery simulation model of engineer[J]. Journal of Aerospace Power, 2019, 34(3): 616-626 (in Chinese)

[6] 刘晓娟, 王华伟, 徐璇. 考虑多退化失效和突发失效之间竞争失效的可靠性评估方法 $[\mathrm{J}]$. 中国机械工程, 2017, 28 (1) : 7-12

LIU Xiaojuan, WANG Huawei, XU Xuan. Reliability assessment based on competition failure considering multi-degradation and catastrophic failure $[\mathrm{J}]$. China Mechanical Engineering, 2017, 28(1): 7-12 (in Chinese)

[7] RAFIEE K, FENG Q, COIT D. Reliability assessment of competing risks with generalized mixed shock models $[\mathrm{J}]$. Reliability Engineering and System Safety, 2017, 159: 1-11

[8] QIU Q, CUI L. Reliability evaluation based on a dependent two-stage failure process with competing failures $[\mathrm{J}]$. Applied Mathematical Modeling, 2018, 64: 699-712

[9] YANG L, ZHANG Y, PENG R. Hybrid preventive maintenance of competing failures under random environment $[\mathrm{J}]$. Reliability Engineering and System Safety, 2018, 174: 130-140

[10] HAO S, YANG J. Dependent competing failure modeling for the gil subject to partial discharge and air leakage with random degradation initiation time $[\mathrm{J}]$. IEEE Trans on Reliability, 2019, 68(3): 1070-1079

[11] 齐佳. 性能退化自恢复产品的相关竞争失效可靠性建模与评估 $[\mathrm{D}]$. 哈尔滨: 哈尔滨理工大学, 2019

QI Jia. Reliability modeling and assessment of dependent competing failure for performance degradation self-recovery products [D]. Harbin: Harbin University of Science and Technology, 2019 (in Chinese) 
[12] 逯程, 徐廷学, 李启超, 等. 竞争失效条件下的装备选择性维修优化方法 [J]. 中国惯性技术学报, 2019, 27(2): 272-280

LU Cheng, XU Tingxue, LI Qichao, et al. Optimal method for selective maintenance of equipment subject to competing failure [J]. Journal of Chinese Inertial Technology, 2019, 27(2) : 272-280 (in Chinese)

[13] SASAK A, YAMAMOT T. A review of studies of hydraulic lock[J]. Lubrication Eng, 1993, 49: 585-593

[14] FAN M, ZENG Z, ZIO E. Modeling dependent competing failure processes with degradation-shock dependence[J]. Reliability Engineering and System Safety, 2017, 165: 422-430

[15] LEE S, GILMORE B. The determination of the probabilistic of velocities acceleration in kinematic chain with uncertainty $[\mathrm{J}]$. Journal of Mechanical Design, 1991, 113(1):84-90

[16] ARCHARD J. Contact and rubbing of flat surfaces[J]. Journal of Applied Physics, 1953, 24: 123-128

\title{
Competing failure analysis of aircraft connecting-rod-type cabin door retraction mechanism with intermission considered
}

\author{
LIU Jingyi ${ }^{1}$, ZHANG Yugang ${ }^{2}$, ZHUANG Xinchen ${ }^{3}$, PANG Huan ${ }^{1}$ \\ (1.School of Automobile, Chang'an University, Xi'an 710064, China; \\ 2.School of Aeronautics, Northwestern Polytechnical University, Xi'an 710072, China ; \\ 3.School of Mechanical Engineering, Tsinghua University, Beijing 100091, China
}

\begin{abstract}
A novel competing failure model considering intermission is established in this paper, which is appropriate for aircraft connecting-rod-type cabin door retraction mechanism. Poisson process, Archard wear model and failure functions are integrated to describe the catastrophic failure and degradation failure of the aircraft mechanism. Intermission is introduced in the novel competing failure model after considering the actual working condition and working factors. Based on the analysis of mechanism working principle and failure characteristics, a certain aircraft connecting-rod-type cabin door retraction mechanism is studied as an example. The case results indicate that this mechanism has an unusual degradation process. Besides, the novel competing failure model with intermission is effective in engineering project.
\end{abstract}

Keywords : competing failures; intermission; aircraft connecting-rod-type cabin door retraction mechanism

引用格式: 刘敬一, 张玉刚, 庄新臣, 等. 考虑间歇期的飞机连杆式舱门收放机构竞争失效分析 $[J]$. 西北工业大学学报, $2021,39(5): 1105-1113$

LIU Jingyi, ZHANG Yugang, ZHUANG Xinchen, et al. Competing failure analysis of aircraft connecting-rod-type cabin door retraction mechanism with intermission considered $[\mathrm{J}]$. Journal of Northwestern Polytechnical University, 2021, 39 (5): 1105-1113 (in Chinese) 